\title{
Aus aller Welt
}

\section{Die postmenopausale vulvo- vaginale Atrophie begünstigt Depression und Angststörung} In einer Fall-Kontroll-Studie an postmenopausalen Frauen $(n=125.889$ Fälle, $n=376.057$ Kontrollen; $\varnothing$ Alter 60,7 Jahre) zeigte sich für Frauen mit vulvovaginaler Atrophie ein signifikant erhöhtes Risiko für die Diagnose einer Depression (23,9\% vs. 18,9\%, $p<0,0001)$ bzw. Angststörung (16,6\% vs. $11,3 \% ; p<0,0001)$. Dieses signifikante Risiko bestand auch nach Adjustierung für sonstige mit der Menopause assoziierte Faktoren (Insomnie, Hitzewallungen, Dysurie und Östrogentherapie).

Fazit: Das Risiko für die Diagnose einer Depression ist v. a. in der Peri 2,5fach höher als in der Prämenopause. Wichtig ist, die symptomatische vulvovaginale Atrophie als kausalen Faktor überhaupt miteinzubeziehen!

Moyneur E et al. (2020) Menopause. 27(2):134-142

\section{Knochengesundheit bei Frauen mit Turner-Syndrom}

Ein frühzeitiger Östrogenmangel erhöht das Risiko für Osteoporose. In einer Querschnittsstudie wurde die Knochengesundheit von Frauen mit prämaturer Ovarialinsuffizienz (POI) $(n=67$, medianes Alter 28,1 Jahre) und Turner-Syndrom (TS) ( $n=267$, medianes Alter 34,3 Jahre) miteinander verglichen. Per Fragebogen wurden u.a. stattgehabte Frakturen (unterteilt in "major osteoporotic fractures" und "other fracture types"), Komorbiditäten, Medikation und Östrogenexposition erfasst. Klinische Parameter waren Körpergrösse und -gewicht, Vitamin-D-Serumspiegel sowie alters- und grössenadjustierte T-Scores der Hüfte und Wirbelsäule. Die Frakturrate war bei Frauen mit TS (30,5\%) und POI (32,8\%) vergleichbar. Im Vergleich zu Frauen mit POI hatten Frauen mit TS jedoch signifikant häufiger "major osteoporotic fractures"
$(30,2 \%$ vs. $52,7 \%, p=, 012)$. Frauen mit TS, die Frakturen erlitten, hatten signifikant häufiger eine Hörminderung im Vergleich zu Frauen mit TS ohne Frakturen $(62,2 \%$ vs. $48,1 \%, p=, 045)$.

Fazit: Bei (jüngeren) Frauen mit TS an Knochengesundheit und Hörfähigkeit denken und überprüfen!

Cardona A et al. (2019) Clin Endocrinol (Oxf). 91(6):743-749

\section{Nebenwirkungsprofil der} anti-östrogenen Therapie bei Frauen mit Mammakarzinom in Abhängigkeit vom Alter

In einer Querschnittsstudie wurden 2044> 55-jährige, anti-östrogen behandelte Frauen mit Brustkrebs per Fragebogen bzgl. 15 (menopausaler) Symptome befragt. In der Altersgruppe 56-69 Jahre wurden 1477 Frauen mit Aromataseinhibitoren (Al) und 123 Frauen mit Selektiven Östrogenrezeptormodulatoren (SERM) behandelt. In der Altersgruppe 70+ erhielten 581 Frauen Al und 51 Frauen SERMs. Bei Frauen 70+ traten unter Al folgende Symptome signifikant häufiger und stärker als unter SERM auf: Schwitzen, Fingergelenksteifigkeit, Knie- und Schulterarthralgien, Taubheitsgefühl der Extremitäten und Lethargie. Bei 56-69-jährigen Frauen traten unter Al signifikant häufiger Fingergelenksteifigkeit und Scheidentrockenheit auf, wohingegen unter SERM signifikant häufiger Hitzewallungen, Leukorrhoe, vaginale Blutung und Gewichtszunahme berichtet wurden.

Fazit: Frauen $70+$ haben unter Al ein facettenreicheres und intensiveres Nebenwirkungsprofil als Frauen mit Al $<70$ Jahren.

Honma N et al. (2019) Support Care Cancer. 27(10):3813-3822.

\section{Dienogest reduziert signifikant primäre Dysmenorrhoe}

In einer randomisierten, Plazebo-kontrollierten Studie erhielten 235 Frauen mit primärer Dysmenorrhoe während
12 Wochen entweder Dienogest (DNG) 0,5 mg/Tag, DNG $1 \mathrm{mg} / \mathrm{Tag}$, DNG 2 mg/ Tag, Ethinylestradiol 0,02 mg/Drospirenon 3 mg („Referenzpille ${ }^{\prime \prime}$ ) oder Plazebo. Unter DNG kam es zu einer signifikanten Abnahme der Dysmenorrhoe, welche für die Dosierungen $1 \mathrm{mg} / \mathrm{Tag}$ bzw. $2 \mathrm{mg} / \mathrm{Tag}$ signifikant stärker als unter der ,Referenzpille“ ausgeprägt war.

Fazit: 0,5-1 Tab./Tag der auf dem Schweizer Markt erhältlichen DNGMonopräparate $(2 \mathrm{mg}$ ) können bei primärer Dysmenorrhoe eingesetzt werden.

Osuga $Y$ et al. (2020) Fertil Steril. 13(1):167-175

\section{DRSP Mono-Kontrazeptivum}

Drospirenon (DRSP) $4 \mathrm{mg}$ wurde unter dem Namen Slynd ${ }^{\circledR} 2019$ von der FDA zur Kontrazeption zugelassen. Anders als der täglich einzunehmende östrogenfreie Ovulationshemmer mit Desogestrel 0,075 mg wird DRSP im 24/4-Regime verwendet. Das Einnahmefenster beträgt 24 Stunden (Desogestrel 12 Stunden). In einer Phase-3-Studie wurde das Blutungsprofil von DRSP $4 \mathrm{mg} 24 / 4$ $(n=858)$ mit Desogestrel 0,075 mg/d ( $n=332$ ) während 9 Monate verglichen. In den ersten 7 Monaten traten unter DRSP signifikant seltener Zwischenblutungen als unter Desogestrel auf.

Fazit: Hoffentlich steht in der Schweiz DRSP mono zur Kontrazeption auch bald zur Verfügung!

Palacios Set al. (2019) Arch Gynecol Obstet. 300(6):1805-1812

Hinweis des Verlags. Der Verlag bleibt in Hinblick auf geografische Zuordnungen und Gebietsbezeichnungen in veröffentlichten Karten und Institutsadressen neutral.

J. Gynäkol. Endokrinol. CH 2020 · 23:37 https://doi.org/10.1007/s41975020-00136-1

(c) Springer-Verlag GmbH Austria, ein Teil von Springer Nature 2020
Autorin: Prof. Dr. Petra Stute, Inselspital Bern 